\title{
NUMERICAL ANALYSIS OF A QUASISTATIC PROBLEM OF SLIDING FRICTIONAL CONTACT WITH WEAR*
}

\author{
JIUHUA CHEN ${ }^{\dagger}$, WEIMIN HAN ${ }^{\ddagger}$, AND MIRCEA SOFONEA ${ }^{\S}$
}

\begin{abstract}
We consider numerical approximations of a quasistatic problem modeling the sliding frictional contact with wear between a viscoelastic body and a rigid moving foundation. The contact is modeled with the Coulomb's law of dry friction and the wear is described by a version of Archard's law. The variational formulation of the problem consists of a nonlinear evolutionary equation coupled with a time-dependent variational inequality with nonlinear differential operators, which has a unique solution under certain assumptions on the given data. We derive error estimates for both spatially semi-discrete and fully discrete schemes to solve the problem. Under appropriate regularity assumptions on the exact solution, we establish optimal order error estimates.
\end{abstract}

1. Introduction. We consider a mathematical model for the process of bilateral frictional contact of a viscoelastic body with a rigid moving foundation, such that there is no lose of contact between the body and the foundation. The framework is that of small displacement and small strain theory. The external time dependent volume forces and tractions are assumed to vary slowly; as a result the mechanical states evolve quasistatically. We assume a sliding frictional contact which involves wear of the contacting surface. The friction is modeled with Coulomb's law and the wear is modeled by a version of Archard's law.

Situations of frictional contact between deformable bodies can be frequently found in industry and everyday life such as train wheels with the rails, a shoe with the floor, tectonic plates, the car's braking system, etc. For this reason, considerable progress has been made with the modeling and analysis of contact problems. An early attempt to study frictional contact problems within the framework of variational inequalities was made in [5]. An excellent reference on analysis and numerical approximations of contact problems involving elastic materials with or without friction is [12]. The mathematical, mechanical and numerical state of the art can be found in the proceedings [18].

Wear is one of the plagues which reduce the lifetime of modern machine elements. It represents the unwanted removal of materials from surfaces of contacting bodies occuring in relative motion. Wear arises when a hard rough surface slides against a softer surface, digs into it, and its asperities plough a series of grooves. When two surfaces come into contact, rearrangement of the surface asperities takes place. When they are in relative motion some of the peaks will break and therefore the harder surface removes the softer material. This phenomenon involves the wear of the contacting surfaces. Material loss of wearing solids, the generation and circulation of free wear debris are the main behaviors of the wear process. The loose particles form a thin wear product layer on the body surface. Tribological experiments show that this layer has a great influence on contact phenomena and the wear particles between sliding surface affect the frictional behavior. Realistically, wear cannot be

\footnotetext{
*Received December 4, 1998; revised February 21, 2001.

†Department of Mathematics, University of Iowa, Iowa City, IA 52242, USA.

$\ddagger$ Department of Mathematics, University of Iowa, Iowa City, IA 52242, USA (whan@math. uiowa.edu).

$\S$ Laboratoire de Théorie des Systèmes, University of Perpignan, France.
} 
totally eliminated. It is very difficult to express accurately a quantitative law for the wear because of the many factors that affect this process. It is known that hard materials wear less than soft and the rate of wear of metals is inversely proportional to the hardness of material $[16,24]$. Very often wear increases with increase of loads and sliding time [24].

Generally, a mathematical theory of friction and wear should be a generalization of experimental facts and it must be in agreement with the laws of thermodynamics of irreversible processes. The first trials of a thermodynamical description of the friction and wear processes were provided in $[3,13,14,15]$. General models of quasistatic frictional contact with wear between deformable bodies were derived in $[22,23]$ from thermodynamic considerations. There a dual pseudo-potential with a general friction and wear limit criterion was investigated, from which Coulomb's law of friction and Archard's law of wear were obtained. The consistency of such models in the case of small displacements and small strain theory was also discussed. The models derived in $[22,23]$ were used in various papers where existence and uniqueness results of weak solutions have been proved. For example, a dynamic thermoelastic contact problem with normal compliance and surface wear has been analysed in [2] and variational analysis in the study of viscoelastic frictional contact problems with wear has been provided in [19, 20, 21].

The present paper represents a continuation of [21]. Its aim is to provide numerical analysis of a quasistatic problem of sliding frictional contact with wear, similar to that studied in [21]. We model the process as in [22, 23] by introducing the wear function which measures the wear of the contact surface and which satisfies Archard's law. The friction is modeled with the sliding version of Coulomb's law. The well-posedness of the problem is stated and may be obtained using the arguments of [21]. In a variational formulation, the problem consists of a nonlinear evolution equation coupled with a time-dependent variational inequality with nonlinear differential operators. The literature is abundant on numerical treatment of variational inequality, see for instance the monographs $[6,7,11,12]$. Of particular relevance to this paper are the works on numerical analysis of variational inequalities arising in plasticity, cf. [8, 9, 10].

The paper is organized as follows. In Section 2 we present the mechanical problem together with its variational formulation. We then list the assumptions on the data and state an existence and uniqueness result, which shows that under a smallness assumption on the given data, the mechanical problem has a unique weak solution. In Sections 3 and 4 we analyze spatially semi-discrete and fully discrete schemes, respectively. We use the finite element method to discretize the spatial domain and a backward Euler scheme to obtain the fully discrete problems. We also derive error estimates for both spatially semi-discrete and fully discrete schemes. Finally, under appropriate regularity assumptions on the exact solution, we obtain optimal order error estimates.

We thank the two referees whose suggestions lead to an improvement of the paper.

2. The problem of sliding frictional contact with wear. In this section we describe a model for the contact problem with wear, present its variational formulation, list the assumptions imposed on the problem data and state an existence and uniqueness result.

The physical setting is as follows. We consider a viscoelastic body whose material particles occupy a bounded domain $\Omega$ of $\mathbb{R}^{d}(d=2,3$ in applications). For the 
domain $\Omega$, we assume that its boundary $\Gamma$ is Lipschitz continuous, and is partitioned into three disjoint measurable parts $\Gamma_{1}, \Gamma_{2}$ and $\Gamma_{3}$, with meas $\left(\Gamma_{1}\right)>0$. Displacement and surface traction conditions will be specified on $\Gamma_{1}$ and $\Gamma_{2}$, respectively. On $\Gamma_{3}$, the body is in frictional bilateral contact with a moving plane foundation, which results in the wear of the contacting surface. We assume that there is only sliding contact which is always maintained. Let $[0, T]$ be the time interval of interest.

As usual, we will use the notation $\boldsymbol{u}=\left(u_{i}\right): \Omega \times[0, T] \rightarrow \mathbb{R}^{d}$ for the displacement field and $\sigma=\left(\sigma_{i j}\right): \Omega \times[0, T] \rightarrow S_{d}$ for the stress field. Here and throughout this paper, the indices $i$ and $j$ run between 1 and $d$, we adopt the summation convention over repeated indices, unless stated otherwise, and the index that follows a comma indicates a partial derivative with respect to the corresponding component of the independent variable. We use $S_{d}$ to represent the space of second order symmetric tensors on $\mathbb{R}^{d}$, or equivalently, the space of symmetric matrices of order $d$. We define the inner products and the corresponding norms on $\mathbb{R}^{d}$ and $S_{d}$ by

$$
\begin{aligned}
& \boldsymbol{u} \cdot \boldsymbol{v}=u_{i} v_{i}, \quad|\boldsymbol{v}|=(\boldsymbol{v} \cdot \boldsymbol{v})^{1 / 2}, \quad \forall \boldsymbol{u}, \boldsymbol{v} \in \mathbb{R}^{d}, \\
& \boldsymbol{\sigma} \cdot \boldsymbol{\tau}=\sigma_{i j} \tau_{i j}, \quad|\boldsymbol{\tau}|=(\boldsymbol{\tau} \cdot \boldsymbol{\tau})^{1 / 2}, \quad \forall \boldsymbol{\sigma}, \boldsymbol{\tau} \in S_{d} .
\end{aligned}
$$

Since the boundary $\Gamma$ is Lipschitz continuous, the unit outward normal vector $\boldsymbol{\nu}$ on the boundary is defined a.e. For every vector field $\boldsymbol{v}$, we use the notation $\boldsymbol{v}$ to denote the trace of $\boldsymbol{v}$ on $\Gamma$ and we denote by $v_{\nu}$ and $\boldsymbol{v}_{\tau}$ the normal and the tangential components of $\boldsymbol{v}$ on the boundary given by

$$
v_{\nu}=\boldsymbol{v} \cdot \boldsymbol{\nu}, \quad \boldsymbol{v}_{\tau}=\boldsymbol{v}-v_{\nu} \boldsymbol{\nu}
$$

We also use the notation $\varepsilon(\boldsymbol{v})$ for the tensor field defined by

$$
\varepsilon(\boldsymbol{v})=\left(\varepsilon_{i j}(\boldsymbol{v})\right), \quad \varepsilon_{i j}(\boldsymbol{v})=\frac{1}{2}\left(v_{i, j}+v_{j, i}\right)
$$

For a stress field $\sigma$, the application of its trace on the boundary to $\nu$ is the Cauchy stress vector $\sigma \nu$. We define, similarly, the normal and tangential components of the stress on the boundary by the formulae

$$
\sigma_{\nu}=(\sigma \nu) \cdot \nu, \quad \sigma_{\tau}=\sigma \nu-\sigma_{\nu} \nu
$$

Finally, in the sequel div will denote the divergence operator for tensor fields, i.e.

$$
\operatorname{div} \boldsymbol{\sigma}=\left(\sigma_{i j, j}\right) \text {. }
$$

The material is assumed to be viscoelastic, its constitutive relation being

$$
\boldsymbol{\sigma}=A(\varepsilon(\dot{\boldsymbol{u}}))+G(\varepsilon(\boldsymbol{u})),
$$

where $A$ and $G$ are given nonlinear constitutive functions and $\boldsymbol{\varepsilon}(\boldsymbol{u})$ represents the small strain tensor. Here and below a dot above a variable represents its time derivative. We recall that in linear viscoelasticity, the stress tensor $\sigma=\left(\sigma_{i j}\right)$ is given by

$$
\sigma_{i j}=a_{i j k l} \varepsilon_{k l}(\dot{u})+g_{i j k l} \varepsilon_{k l}(u)
$$

where $A=\left(a_{i j k l}\right)$ is the viscosity tensor and $G=\left(g_{i j k l}\right)$ is the elasticity tensor, for $i, j, k, l=1, \ldots, d$. Kelvin-Voigt viscoelastic materials of the form (2.1) involving nonlinear constitutive functions have been considered recently in $[19,20]$. 
We assume that the viscoelastic body is fixed on $\Gamma_{1}$, and therefore the displacement field vanishes there. We also assume that a body force of density $f$ acts in $\Omega$, and surface traction of density $\boldsymbol{g}$ is imposed on $\Gamma_{2}$. The densities $f$ and $\boldsymbol{g}$ may depend on the time variable and are assumed to vary slowly in time; the accelerations are therefore neglected in the equations of motion, leading to a quasistatic approach of the process.

We now briefly describe the boundary conditions on the contact surface $\Gamma_{3}$, using the model derived in $[22,23]$. We introduce the wear function $w: \Gamma_{3} \times[0, T] \rightarrow \mathbb{R}$ which measures the wear of the surface. The wear is identified as an increase in gap in the normal direction between the body and the foundation or, equivalently, as the normal depth of the material that is lost. Since the body is in bilateral contact with the foundation it follows that

$$
u_{\nu}=-w
$$

on $\Gamma_{3}$. Thus the position of the contact evolves with the wear. We remark that the effect of the wear is the recession on $\Gamma_{3}$ and therefore is expected that $u_{\nu}<0$ on $\Gamma_{3}$ which implies $w>0$ on $\Gamma_{3}$. We conclude that the wear is positive which justify the sign convention in (2.2).

The evolution of the wear of the contacting surface is governed by a simplified version of Archard's law (cf. [22, 23]) which we now describe. The rate form of Archard's law is

$$
\dot{w}=-k_{w} \sigma_{\nu}\left|\dot{u}_{\tau}-v^{*}\right|
$$

where $k_{w}>0$ is a wear coefficient, $\boldsymbol{v}^{*}$ is the velocity of the foundation, and $\left|\dot{\boldsymbol{u}}_{\tau}-\boldsymbol{v}^{*}\right|$ represents the slip between the contact surface and the foundation. We see that the rate of wear is assumed to be proportional to the contact stress and the slip. For the sake of simplicity we assume in the sequel that the motion of the foundation is uniform, i.e. $v^{*}$ is a constant vector in the plane of the foundation and we denote $v^{*}=\left|\boldsymbol{v}^{*}\right|>0$. We also assume that $v^{*}$ is large and therefore we neglect in the sequel $\dot{u}_{\tau}$ as compared with $\boldsymbol{v}^{*}$ to obtain the following version of Archard's law

$$
\dot{w}=-k_{w} \sigma_{\nu} v^{*} \text {. }
$$

Use of the simplified law (2.3) for the evolution of the wear avoids some mathematical difficulties in the study of the quasistatic viscoelastic problem.

We can now eliminate the unknown function $w$ from our problem. Let $\alpha=k_{w} v^{*}$ and $\beta=1 / \alpha$. Using (2.2) and (2.3) we have

$$
\sigma_{\nu}=\beta \dot{u}_{\nu}
$$

We model the frictional contact between the viscoelastic body and the foundation with Coulomb's law of dry friction. Since there is only sliding contact it follows that

$$
\left|\sigma_{\tau}\right|=\mu\left|\sigma_{\nu}\right|, \quad \sigma_{\tau}=-\lambda\left(\dot{u}_{\tau}-\boldsymbol{v}^{*}\right), \quad \lambda \geq 0
$$

where $\mu>0$ is the coefficient of friction. These equalities show that the tangential stress is limited and it is in the opposite direction to the relative velocity $\dot{\boldsymbol{u}}_{\tau}-\boldsymbol{v}^{*}$.

Moreover, the wear increases in time, i.e. $\dot{w} \geq 0$ and therefore, it follows from (2.2) and (2.3) that $\dot{u}_{\nu} \leq 0$ and $\sigma_{\nu} \leq 0$ on $\Gamma_{3}$. Thus, the conditions (2.4) and (2.5) imply

$$
-\sigma_{\nu}=\beta\left|\dot{u}_{\nu}\right|, \quad\left|\sigma_{\tau}\right|=-\mu \sigma_{\nu}, \quad \sigma_{\tau}=-\lambda\left(\dot{u}_{\tau}-\boldsymbol{v}^{*}\right), \quad \lambda \geq 0
$$


on $\Gamma_{3}$. Using now (2.1) as constitutive law and (2.6) as contact conditions, the classical formulation of the mechanical problem of sliding frictional contact with wear is the following: Find a displacement $\boldsymbol{u}: \Omega \times[0, T] \rightarrow \mathbb{R}^{d}$ and a stress tensor $\boldsymbol{\sigma}: \Omega \times[0, T] \rightarrow$ $S_{d}$ such that

$$
\left\{\begin{array}{l}
\boldsymbol{\sigma}=A(\varepsilon(\dot{u}))+G(\varepsilon(u)) \text { in } \Omega \times(0, T) \\
\operatorname{div} \boldsymbol{\sigma}+\boldsymbol{f}=\mathbf{0} \text { in } \Omega \times(0, T) \\
\boldsymbol{u}=\mathbf{0} \text { on } \Gamma_{1} \times(0, T) \\
\boldsymbol{\sigma} \boldsymbol{\nu}=\boldsymbol{g} \text { on } \Gamma_{2} \times(0, T) \\
-\sigma_{\nu}=\beta\left|\dot{u}_{\nu}\right|,\left|\boldsymbol{\sigma}_{\tau}\right|=-\mu \sigma_{\nu}, \boldsymbol{\sigma}_{\tau}=-\lambda\left(\dot{\boldsymbol{u}}_{\tau}-\boldsymbol{v}^{*}\right), \lambda \geq 0 \quad \text { on } \Gamma_{3} \times(0, T) \\
\boldsymbol{u}(0)=u_{0} \text { in } \Omega
\end{array}\right.
$$

Here $u_{0}$ represents the given initial displacement.

To present the variational formulation of this problem we need to introduce some functional notation. For the displacement variable, we use the space

$$
V=\left\{\boldsymbol{v}=\left(v_{i}\right) \in\left(H^{1}(\Omega)\right)^{d}: \boldsymbol{v}=\mathbf{0} \text { on } \Gamma_{1}\right\}
$$

with the canonical inner product defined by

$$
\langle\boldsymbol{u}, \boldsymbol{v}\rangle_{V}=\int_{\Omega} u_{i} v_{i} d x+\int_{\Omega} \varepsilon_{i j}(u) \varepsilon_{i j}(\boldsymbol{v}) d x
$$

and the associate norm

$$
\|v\|_{V}=\sqrt{\langle v, v\rangle_{V}}
$$

For stress and strain fields, we use the space

$$
Q=\left\{\tau \in\left(L^{2}(\Omega)\right)^{d \times d}: \tau_{i j}=\tau_{j i}, 1 \leq i, j \leq d\right\}
$$

with the canonical inner product defined by

$$
\langle\tau, \xi\rangle_{Q}=\int_{\Omega} \tau_{i j} \xi_{i j} d x
$$

and the corresponding norm defined by

$$
\|\tau\|_{Q}^{2}=\langle\tau, \tau\rangle
$$

We will also need the space

$$
H(\operatorname{div} ; \Omega)=\left\{\boldsymbol{\tau} \in Q: \operatorname{div} \boldsymbol{\tau} \in\left(L^{2}(\Omega)\right)^{d}\right\} .
$$

Since meas $\left(\Gamma_{1}\right)>0$, Korn's inequality holds $([17])$ :

$$
\|\boldsymbol{v}\|_{V} \leq c\|\varepsilon(\boldsymbol{v})\|_{Q} \quad \forall \boldsymbol{v} \in V
$$

where $c$ is a positive constant depending on $\Omega$ and $\Gamma_{1}$. Everywhere in this paper, the symbol $c$ will represent a positive constant which may change its value from place to place, and may depend on the input data, but independent of discretization parameters $h$ and $k$ to be introduced later.

From (2.8) it follows that $\boldsymbol{v} \mapsto\|\varepsilon(\boldsymbol{v})\|_{Q}$ is a norm over the space $V$, equivalent to the norm $\boldsymbol{v} \mapsto\|\boldsymbol{v}\|_{V}$. 
For any normed space $X, C([0, T] ; X)$ denotes the space of continuous functions from $[0, T]$ to $X$, with the norm

$$
\|f\|_{C([0, T] ; X)}=\max _{t \in[0, T]}\|f(t)\|_{X} .
$$

Similarly, $C^{1}([0, T] ; X)$ denotes the space of continuously differentiable functions from $[0, T]$ to $X$, with the norm

$$
\|f\|_{C^{1}([0, T] ; X)}=\max _{t \in[0, T]}\|f(t)\|_{X}+\max _{t \in[0, T]}\|\dot{f}(t)\|_{X} .
$$

When $X$ is a Banach space, both $C([0, T] ; X)$ and $C^{1}([0, T] ; X)$ are Banach spaces.

In the study of the mechanical problem (2.7) we make the following assumptions.

(a) $A: \Omega \times S_{d} \rightarrow S_{d}$.

(b) There exists $\mathcal{L}_{A}>0$ such that

$\left|A\left(\boldsymbol{x}, \varepsilon_{1}\right)-A\left(\boldsymbol{x}, \varepsilon_{2}\right)\right| \leq \mathcal{L}_{A}\left|\varepsilon_{1}-\varepsilon_{2}\right| \quad \forall \varepsilon_{1}, \varepsilon_{1} \in S_{d}$, a.e. $\boldsymbol{x} \in \Omega$.

(c) There exists $m>0$ such that

$\left(A\left(x, \varepsilon_{1}\right)-A\left(x, \varepsilon_{2}\right)\right) \cdot\left(\varepsilon_{1}-\varepsilon_{2}\right) \geq m\left|\varepsilon_{1}-\varepsilon_{2}\right|^{2} \quad \forall \varepsilon_{1}, \varepsilon_{1} \in S_{d}$, a.e. $x \in \Omega$.

(d) For any $\varepsilon \in S_{d}, \boldsymbol{x} \mapsto A(\boldsymbol{x}, \varepsilon)$ is Lebesgue measurable on $\Omega$.

(e) The mapping $\boldsymbol{x} \mapsto A(\boldsymbol{x}, \mathbf{0}) \in Q$.

(a) $G: \Omega \times S_{d} \rightarrow S_{d}$.

(b) There exists an $\mathcal{L}_{G}>0$ such that $\left|G\left(\boldsymbol{x}, \varepsilon_{1}\right)-G\left(\boldsymbol{x}, \varepsilon_{2}\right)\right| \leq \mathcal{L}_{G}\left|\varepsilon_{1}-\varepsilon_{2}\right| \quad \forall \varepsilon_{1}, \varepsilon_{2} \in S_{d}$, a.e. $\boldsymbol{x} \in \Omega,$.

(c) For any $\varepsilon \in S_{d}, \boldsymbol{x} \mapsto G(\boldsymbol{x}, \varepsilon)$ is measurable.

(d) The mapping $\boldsymbol{x} \mapsto G(\boldsymbol{x}, \mathbf{0}) \in Q$.

(2.11 $\left\{\begin{array}{l}\boldsymbol{f} \in C\left([0, T] ;\left(L^{2}(\Omega)\right)^{d}\right), \\ \boldsymbol{g} \in C\left([0, T] ;\left(L^{2}\left(\Gamma_{2}\right)\right)^{d}\right), \\ \mu \in L^{\infty}\left(\Gamma_{3}\right), \quad \mu(\boldsymbol{x}) \geq 0 \text { a.e. on } \Gamma_{3}, \\ \beta \in L^{\infty}\left(\Gamma_{3}\right), \quad \beta(\boldsymbol{x}) \geq \beta_{*}>0 \text { a.e. on } \Gamma_{3}, \\ u_{0} \in V .\end{array}\right.$

We define the functionals

$$
\begin{aligned}
& L(t ; \boldsymbol{v})=\int_{\Omega} \boldsymbol{f}(t) \cdot \boldsymbol{v} d x+\int_{\Gamma_{2}} \boldsymbol{g}(t) \cdot \boldsymbol{v} d s \\
& j(\boldsymbol{u}, \boldsymbol{v})=\int_{\Gamma_{3}} \beta\left|u_{\nu}\right|\left(\mu\left|\boldsymbol{v}_{\tau}-\boldsymbol{v}^{*}\right|+v_{\nu}\right) d s
\end{aligned}
$$

for all $\boldsymbol{u}, \boldsymbol{v} \in V$ and $t \in[0, T]$, where $d s$ denotes the surface element.

The variational formulation of the mechanical problem (2.7) can be stated as follows.

Problem P. Find a displacement $\boldsymbol{u}: \Omega \times[0, T] \rightarrow \mathbb{R}^{d}$ and a stress field $\boldsymbol{\sigma}$ : $\Omega \times[0, T] \rightarrow S_{d}$, such that for $t \in[0, T]$,

(2.12) $\boldsymbol{\sigma}(t)=A(\varepsilon(\dot{u}(t)))+G(\varepsilon(\boldsymbol{u}(t)))$ in $\Omega$,

$(2.13)\langle\boldsymbol{\sigma}(t), \boldsymbol{\varepsilon}(\boldsymbol{v}-\dot{\boldsymbol{u}}(t))\rangle+j(\dot{\boldsymbol{u}}(t), \boldsymbol{v})-j(\dot{\boldsymbol{u}}(t), \dot{\boldsymbol{u}}(t)) \geq L(t ; \boldsymbol{v}-\dot{\boldsymbol{u}}(t)) \quad \forall \boldsymbol{v} \in V$,

and the initial condition

$$
\boldsymbol{u}(0)=u_{0}
$$


Well-posedness of this problem is given by the the following result.

THEOREM 2.1. Let the assumptions (2.9)-(2.11) hold. Then there is a constant $\alpha_{0}>0$ which depends only on $\Omega, \Gamma_{1}, \Gamma_{3}$ and $A$, such that if

$$
\|\beta\|_{L^{\infty}\left(\Gamma_{3}\right)}\left(\|\mu\|_{L^{\infty}\left(\Gamma_{3}\right)}+1\right)<\alpha_{0},
$$

then the problem $\mathbf{P}$ has a unique solution $\boldsymbol{u} \in C^{1}([0, T] ; V), \boldsymbol{\sigma} \in C([0, T] ; H(\operatorname{div} ; \Omega))$.

The proof of Theorem 2.1 is carried out in several steps, using the same arguments as in [21]. Since the modifications are straightforward, we omit here the details. We just recall that the proof is based on classical results for elliptic variational inequalities followed by fixed point arguments. In the rest of the paper, we assume the conditions stated in Theorem 2.1 are satisfied so that the contact problem $\mathbf{P}$ has a unique solution.

We end this section with the remark that if $v^{*}$ is large enough then $\beta=1 /\left(k_{w} v^{*}\right)$ is small enough and therefore condition (2.15) which guarantees the unique solvability of problem $\mathbf{P}$ is satisfied. We conclude that the mechanical problem (2.7) has a unique weak solution if the velocity of the foundation is large enough.

3. Spatially semi-discrete approximation. In this section we consider an approximation of the problem $\mathbf{P}$ by discretizing only the spatial domain. Let $V^{h} \subset V$ and $Q^{h} \subset Q$ be finite-dimensional spaces which for example, can be constructed by the finite element method. We assume that these spaces satisfy

$$
\varepsilon\left(V^{h}\right) \subset Q^{h} .
$$

This assumption is very natural and is valid when the polynomial degree for the space $V^{h}$ is at most one higher than that for the space $Q^{h}$.

Let $\mathcal{P}_{Q^{h}}: Q \rightarrow Q^{h}$ be the orthogonal projection defined through the relation

$$
\left\langle\mathcal{P}_{Q^{h}} \boldsymbol{q}, \boldsymbol{q}^{h}\right\rangle_{Q}=\left\langle\boldsymbol{q}, \boldsymbol{q}^{h}\right\rangle_{Q} \quad \forall \boldsymbol{q} \in Q, \boldsymbol{q}^{h} \in Q^{h}
$$

Obviously, we have

$$
\left\|\mathcal{P}_{Q^{h}} \boldsymbol{q}\right\|_{Q} \leq\|\boldsymbol{q}\|_{Q} \quad \forall \boldsymbol{q} \in Q
$$

This property will be used on various occasions.

We now discuss a spatially semi-discrete scheme.

Problem $\mathbf{P}^{h}$. Find the displacement field $\boldsymbol{u}^{h}:[0, T] \rightarrow V^{h}$ and the stress field $\sigma^{h}:[0, T] \rightarrow Q^{h}$ such that

$$
\boldsymbol{u}^{h}(0)=\boldsymbol{u}_{0}^{h}
$$

and for $t \in[0, T]$,

$$
\begin{aligned}
& \boldsymbol{\sigma}^{h}(t)=\mathcal{P}_{Q^{h}} A\left(\varepsilon\left(\dot{\boldsymbol{u}}^{h}(t)\right)\right)+\mathcal{P}_{Q^{h}} G\left(\boldsymbol{\varepsilon}\left(\boldsymbol{u}^{h}(t)\right)\right) \quad \text { in } \Omega \\
& \left\langle\boldsymbol{\sigma}^{h}(t), \boldsymbol{\varepsilon}\left(\boldsymbol{v}^{h}-\dot{\boldsymbol{u}}^{h}(t)\right)\right\rangle+j\left(\dot{\boldsymbol{u}}^{h}(t), \boldsymbol{v}^{h}\right)-j\left(\dot{\boldsymbol{u}}^{h}(t), \dot{\boldsymbol{u}}^{h}(t)\right) \\
& \quad \geq L\left(t ; \boldsymbol{v}^{h}-\dot{\boldsymbol{u}}^{h}(t)\right) \quad \forall \boldsymbol{v}^{h} \in V^{h} .
\end{aligned}
$$

Here, $\boldsymbol{u}_{0}^{h} \in V^{h}$ is an appropriate approximation of $\boldsymbol{u}_{0}$. 
Using the arguments in [21], it can be shown that under the conditions stated in Theorem 2.1, the problem $\mathbf{P}^{h}$ has a unique solution $\boldsymbol{u}^{h} \in C^{1}\left([0, T] ; V^{h}\right), \boldsymbol{\sigma}^{h} \in$ $C\left([0, T] ; Q^{h}\right)$. Our main purpose here is to derive estimates for the errors $\boldsymbol{u}-\boldsymbol{u}^{h}$ and $\boldsymbol{\sigma}-\boldsymbol{\sigma}^{h}$.

To this end, let $t \in[0, T]$. From (2.12) and (3.4), we have

$$
\begin{aligned}
& \boldsymbol{\sigma}(t)-\boldsymbol{\sigma}^{h}(t) \\
= & \left(I_{Q}-\mathcal{P}_{Q^{h}}\right) \boldsymbol{\sigma}(t)+\mathcal{P}_{Q^{h}}\left[A(\varepsilon(\dot{u}(t)))-A\left(\varepsilon\left(\dot{u}^{h}(t)\right)\right)+G(\varepsilon(\boldsymbol{u}(t)))-G\left(\varepsilon\left(\boldsymbol{u}^{h}(t)\right)\right)\right],
\end{aligned}
$$

where $I_{Q}: Q \rightarrow Q$ is the identity operator.

Using the conditions (2.9), (2.10) and the property (3.2), we obtain

$$
\begin{aligned}
& \left\|\boldsymbol{\sigma}(t)-\boldsymbol{\sigma}^{h}(t)\right\|_{Q} \\
\leq & \left\|\left(I_{Q}-\mathcal{P}_{Q^{h}}\right) \boldsymbol{\sigma}(t)\right\|_{Q}+c\left(\left\|\varepsilon\left(\dot{\boldsymbol{u}}(t)-\dot{\boldsymbol{u}}^{h}(t)\right)\right\|_{Q}+\left\|\varepsilon\left(\boldsymbol{u}(t)-\boldsymbol{u}^{h}(t)\right)\right\|_{Q}\right) .
\end{aligned}
$$

Since

$$
\varepsilon\left(\boldsymbol{u}(t)-\boldsymbol{u}^{h}(t)\right)=\boldsymbol{\varepsilon}\left(\boldsymbol{u}_{0}-\boldsymbol{u}_{0}^{h}\right)+\int_{0}^{t} \varepsilon\left(\dot{\boldsymbol{u}}(r)-\dot{\boldsymbol{u}}^{h}(r)\right) d r
$$

we have

$$
\left\|\varepsilon\left(\boldsymbol{u}(t)-\boldsymbol{u}^{h}(t)\right)\right\|_{Q} \leq\left\|\varepsilon\left(\boldsymbol{u}_{0}-\boldsymbol{u}_{0}^{h}\right)\right\|_{Q}+\int_{0}^{t}\left\|\varepsilon\left(\dot{u}(r)-\dot{u}^{h}(r)\right)\right\|_{Q} d r
$$

Thus from (3.6),

$$
\begin{aligned}
\left\|\boldsymbol{\sigma}(t)-\boldsymbol{\sigma}^{h}(t)\right\|_{Q} \leq & \left\|\left(I_{Q}-\mathcal{P}_{Q^{h}}\right) \boldsymbol{\sigma}(t)\right\|_{Q}+c\left(\left\|\varepsilon\left(\dot{\boldsymbol{u}}(t)-\dot{\boldsymbol{u}}^{h}(t)\right)\right\|_{Q}+\left\|\varepsilon\left(\boldsymbol{u}_{0}-\boldsymbol{u}_{0}^{h}\right)\right\|_{Q}\right) \\
& +c \int_{0}^{t}\left\|\varepsilon\left(\dot{\boldsymbol{u}}(r)-\dot{\boldsymbol{u}}^{h}(r)\right)\right\|_{Q} d r
\end{aligned}
$$

Recalling Korn's inequality (2.8), we conclude

$$
\begin{aligned}
& \left\|\boldsymbol{\sigma}-\boldsymbol{\sigma}^{h}\right\|_{C([0, T] ; Q)} \\
\leq & \left\|\left(I_{Q}-\mathcal{P}_{Q^{h}}\right) \boldsymbol{\sigma}\right\|_{C([0, T] ; Q)}+c\left(\left\|\dot{u}-\dot{u}^{h}\right\|_{C([0, T] ; V)}+\left\|u_{0}-u_{0}^{h}\right\|_{V}\right) .
\end{aligned}
$$

It remains to estimate $\left\|\dot{\boldsymbol{u}}-\dot{\boldsymbol{u}}^{h}\right\|_{C([0, T] ; V)}$. We take $\boldsymbol{v}=\dot{\boldsymbol{u}}^{h}(t)$ in (2.13) and use (2.12) to obtain

$$
\begin{aligned}
& \left\langle A(\varepsilon(\dot{\boldsymbol{u}}(t)))+G(\varepsilon(\boldsymbol{u}(t))), \varepsilon\left(\dot{\boldsymbol{u}}^{h}(t)-\dot{\boldsymbol{u}}(t)\right)\right\rangle+j\left(\dot{\boldsymbol{u}}(t), \dot{\boldsymbol{u}}^{h}(t)\right)-j(\dot{\boldsymbol{u}}(t), \dot{\boldsymbol{u}}(t)) \\
\geq & L\left(t ; \dot{\boldsymbol{u}}^{h}(t)-\dot{\boldsymbol{u}}(t)\right) .
\end{aligned}
$$

Using (3.4) and (3.5), we get

$$
\begin{aligned}
& \left\langle A\left(\varepsilon\left(\dot{\boldsymbol{u}}^{h}(t)\right)\right)+G\left(\boldsymbol{\varepsilon}\left(\boldsymbol{u}^{h}(t)\right)\right), \boldsymbol{\varepsilon}\left(\boldsymbol{v}^{h}-\dot{\boldsymbol{u}}^{h}(t)\right)\right\rangle+j\left(\dot{\boldsymbol{u}}^{h}(t), \boldsymbol{v}^{h}\right)-j\left(\dot{\boldsymbol{u}}^{h}(t), \dot{\boldsymbol{u}}^{h}(t)\right) \\
\geq & L\left(t ; \boldsymbol{v}^{h}-\dot{\boldsymbol{u}}^{h}(t)\right) .
\end{aligned}
$$

Adding these two inequalities and performing some elementary manipulations, we obtain

$$
\begin{aligned}
& \left\langle A(\varepsilon(\dot{\boldsymbol{u}}(t)))-A\left(\varepsilon\left(\dot{\boldsymbol{u}}^{h}(t)\right)\right), \varepsilon\left(\dot{\boldsymbol{u}}(t)-\dot{\boldsymbol{u}}^{h}(t)\right)\right\rangle \\
\leq & \left\langle A(\varepsilon(\dot{\boldsymbol{u}}(t)))-A\left(\varepsilon\left(\dot{\boldsymbol{u}}^{h}(t)\right)\right), \boldsymbol{\varepsilon}\left(\dot{\boldsymbol{u}}(t)-\boldsymbol{v}^{h}\right)\right\rangle \\
& +\left\langle G(\varepsilon(\boldsymbol{u}(t)))-G\left(\varepsilon\left(\boldsymbol{u}^{h}(t)\right)\right), \boldsymbol{\varepsilon}\left(\dot{\boldsymbol{u}}(t)-\boldsymbol{v}^{h}\right)\right\rangle \\
& -\left\langle G(\varepsilon(\boldsymbol{u}(t)))-G\left(\varepsilon\left(\boldsymbol{u}^{h}(t)\right)\right), \boldsymbol{\varepsilon}\left(\dot{\boldsymbol{u}}(t)-\dot{\boldsymbol{u}}^{h}(t)\right)\right\rangle \\
& +R\left(t ; \dot{u}(t), \boldsymbol{v}^{h}\right)+D\left(\dot{\boldsymbol{u}}(t), \dot{\boldsymbol{u}}^{h}(t), \boldsymbol{v}^{h}\right)
\end{aligned}
$$


where

$$
\begin{aligned}
& R\left(t ; \dot{\boldsymbol{u}}(t), \boldsymbol{v}^{h}\right) \\
= & \left\langle\boldsymbol{\sigma}(t), \boldsymbol{\varepsilon}\left(\boldsymbol{v}^{h}-\dot{\boldsymbol{u}}(t)\right)\right\rangle+j\left(\dot{\boldsymbol{u}}(t), \boldsymbol{v}^{h}\right)-j(\dot{\boldsymbol{u}}(t), \dot{\boldsymbol{u}}(t))-L\left(t ; \boldsymbol{v}^{h}-\dot{\boldsymbol{u}}(t)\right), \\
& D\left(\dot{\boldsymbol{u}}(t), \dot{\boldsymbol{u}}^{h}(t), \boldsymbol{v}^{h}\right) \\
= & j\left(\dot{\boldsymbol{u}}(t), \dot{\boldsymbol{u}}^{h}(t)\right)-j\left(\dot{\boldsymbol{u}}(t), \boldsymbol{v}^{h}\right)+j\left(\dot{\boldsymbol{u}}^{h}(t), \boldsymbol{v}^{h}\right)-j\left(\dot{\boldsymbol{u}}^{h}(t), \dot{\boldsymbol{u}}^{h}(t)\right) .
\end{aligned}
$$

Using the assumptions (2.9) and (2.10) on the functions $A$ and $G$, we then have

$$
\begin{aligned}
\left\|\varepsilon\left(\dot{\boldsymbol{u}}(t)-\dot{\boldsymbol{u}}^{h}(t)\right)\right\|_{Q}^{2} \leq & c\left(\left\|\varepsilon\left(\dot{\boldsymbol{u}}(t)-\boldsymbol{v}^{h}\right)\right\|_{Q}^{2}+\left\|\varepsilon\left(\boldsymbol{u}(t)-\boldsymbol{u}^{h}(t)\right)\right\|_{Q}^{2}\right) \\
& +c\left(\left|R\left(t ; \dot{\boldsymbol{u}}(t), \boldsymbol{v}^{h}\right)\right|+\left|D\left(\dot{\boldsymbol{u}}(t), \dot{\boldsymbol{u}}^{h}(t), \boldsymbol{v}^{h}\right)\right|\right),
\end{aligned}
$$

or equivalently,

$$
\begin{aligned}
\left\|\varepsilon\left(\dot{\boldsymbol{u}}(t)-\dot{\boldsymbol{u}}^{h}(t)\right)\right\|_{Q} \leq & c\left(\left\|\varepsilon\left(\dot{\boldsymbol{u}}(t)-\boldsymbol{v}^{h}\right)\right\|_{Q}+\left\|\varepsilon\left(\boldsymbol{u}(t)-\boldsymbol{u}^{h}(t)\right)\right\|_{Q}\right) \\
& +c\left(\left|R\left(t ; \dot{\boldsymbol{u}}(t), \boldsymbol{v}^{h}\right)\right|^{1 / 2}+\left|D\left(\dot{\boldsymbol{u}}(t), \dot{\boldsymbol{u}}^{h}(t), \boldsymbol{v}^{h}\right)\right|^{1 / 2}\right) .
\end{aligned}
$$

Recalling again Korn's inequality (2.8) and the inequality (3.7), we obtain

$$
\begin{aligned}
1)\left\|\dot{\boldsymbol{u}}(t)-\dot{\boldsymbol{u}}^{h}(t)\right\|_{V} \leq & c\left(\left\|\dot{\boldsymbol{u}}(t)-\boldsymbol{v}^{h}\right\|_{V}+\left\|\boldsymbol{u}_{0}-\boldsymbol{u}_{0}^{h}\right\|_{V}+\int_{0}^{t}\left\|\dot{\boldsymbol{u}}(r)-\dot{\boldsymbol{u}}^{h}(r)\right\|_{V} d r\right) \\
& +c\left(\left|R\left(t ; \dot{\boldsymbol{u}}(t), \boldsymbol{v}^{h}\right)\right|^{1 / 2}+\left|D\left(\dot{\boldsymbol{u}}(t), \dot{\boldsymbol{u}}^{h}(t), \boldsymbol{v}^{h}\right)\right|^{1 / 2}\right) .
\end{aligned}
$$

From the trace theorem $V \hookrightarrow\left(L^{2}(\Gamma)\right)^{d}$ (cf. [1]), we have

$$
\|\boldsymbol{v}\|_{\left(L^{2}\left(\Gamma_{3}\right)\right)^{d}} \leq\|\boldsymbol{v}\|_{\left(L^{2}(\Gamma)\right)^{d}} \leq c\|\boldsymbol{v}\|_{V} \quad \forall \boldsymbol{v} \in V .
$$

Now we are ready to estimate the term

$$
D\left(\dot{\boldsymbol{u}}(t), \dot{\boldsymbol{u}}^{h}(t), \boldsymbol{v}^{h}\right)=\int_{\Gamma_{3}} \beta\left(\left|\dot{u}_{\nu}(t)\right|-\left|\dot{u}_{\nu}^{h}(t)\right|\right)\left(\mu\left(\left|\dot{\boldsymbol{u}}_{\tau}^{h}(t)-\boldsymbol{v}^{*}\right|-\left|\boldsymbol{v}_{\tau}^{h}-\boldsymbol{v}^{*}\right|\right)+\dot{u}_{\nu}^{h}(t)-v_{\nu}^{h}\right) d s .
$$

Since

$$
\begin{aligned}
& \left|D\left(\dot{\boldsymbol{u}}(t), \dot{\boldsymbol{u}}^{h}(t), \boldsymbol{v}^{h}\right)\right| \\
\leq & \|\beta\|_{L^{\infty}\left(\Gamma_{3}\right)}\left\|\dot{u}_{\nu}(t)-\dot{u}_{\nu}^{h}(t)\right\|_{L^{2}\left(\Gamma_{3}\right)} \\
& \cdot\left(\|\mu\|_{L^{\infty}\left(\Gamma_{3}\right)}\left\|\dot{\boldsymbol{u}}_{\tau}^{h}(t)-\boldsymbol{v}_{\tau}^{h}\right\|_{\left(L^{2}\left(\Gamma_{3}\right)\right)^{d}}+\left\|\dot{u}_{\nu}^{h}(t)-v_{\nu}^{h}\right\|_{L^{2}\left(\Gamma_{3}\right)}\right) \\
\leq & \|\beta\|_{L^{\infty}\left(\Gamma_{3}\right)}\left(\|\mu\|_{L^{\infty}\left(\Gamma_{3}\right)}+1\right)\left\|\dot{\boldsymbol{u}}(t)-\dot{\boldsymbol{u}}^{h}(t)\right\|_{\left(L^{2}\left(\Gamma_{3}\right)\right)^{d}}\left\|\dot{\boldsymbol{u}}^{h}(t)-\boldsymbol{v}^{h}\right\|_{\left(L^{2}\left(\Gamma_{3}\right)\right)^{d}} \\
\leq & \|\beta\|_{L^{\infty}\left(\Gamma_{3}\right)}\left(\|\mu\|_{L^{\infty}\left(\Gamma_{3}\right)}+1\right)\left\|\dot{\boldsymbol{u}}(t)-\dot{\boldsymbol{u}}^{h}(t)\right\|_{\left(L^{2}\left(\Gamma_{3}\right)\right)^{d}} \\
& \cdot\left(\left\|\dot{\boldsymbol{u}}^{h}(t)-\dot{\boldsymbol{u}}(t)\right\|_{\left(L^{2}\left(\Gamma_{3}\right)\right)^{d}}+\left\|\dot{\boldsymbol{u}}(t)-\boldsymbol{v}^{h}\right\|_{\left.\left(L^{2}\left(\Gamma_{3}\right)\right)^{d}\right)}\right.
\end{aligned}
$$

using the trace theorem (3.12), we have

$$
\begin{aligned}
& \left|D\left(\dot{\boldsymbol{u}}(t), \dot{\boldsymbol{u}}^{h}(t), \boldsymbol{v}^{h}\right)\right| \\
\leq & c\|\beta\|_{L^{\infty}\left(\Gamma_{3}\right)}\left(\|\mu\|_{L^{\infty}\left(\Gamma_{3}\right)}+1\right)\left\|\dot{\boldsymbol{u}}(t)-\dot{\boldsymbol{u}}^{h}(t)\right\|_{V}\left(\left\|\dot{\boldsymbol{u}}^{h}(t)-\dot{\boldsymbol{u}}(t)\right\|_{V}+\left\|\dot{\boldsymbol{u}}(t)-\boldsymbol{v}^{h}\right\|_{V}\right) .
\end{aligned}
$$

Therefore, it follows that

$$
\begin{aligned}
& \left|D\left(\dot{\boldsymbol{u}}(t), \dot{\boldsymbol{u}}^{h}(t), \boldsymbol{v}^{h}\right)\right| \\
\leq & c\|\beta\|_{L^{\infty}\left(\Gamma_{3}\right)}\left(\|\mu\|_{L^{\infty}\left(\Gamma_{3}\right)}+1\right)\left\|\dot{\boldsymbol{u}}(t)-\dot{\boldsymbol{u}}^{h}(t)\right\|_{V}^{2}+c\left\|\dot{\boldsymbol{u}}(t)-\boldsymbol{v}^{h}\right\|_{V}^{2} .
\end{aligned}
$$


Using (3.13) in (3.11), we obtain

$$
\begin{aligned}
& \left\|\dot{\boldsymbol{u}}(t)-\dot{\boldsymbol{u}}^{h}(t)\right\|_{V} \leq c\|\beta\|_{L^{\infty}\left(\Gamma_{3}\right)}^{1 / 2}\left(\|\mu\|_{L^{\infty}\left(\Gamma_{3}\right)}+1\right)^{1 / 2}\left\|\dot{\boldsymbol{u}}(t)-\dot{\boldsymbol{u}}^{h}(t)\right\|_{V} \\
& \quad+c\left(\left\|\dot{\boldsymbol{u}}(t)-\boldsymbol{v}^{h}\right\|_{V}+\left\|\boldsymbol{u}_{0}-\boldsymbol{u}_{0}^{h}\right\|_{V}+\int_{0}^{t}\left\|\dot{\boldsymbol{u}}(r)-\dot{\boldsymbol{u}}^{h}(r)\right\|_{V} d r+\left|R\left(t ; \dot{\boldsymbol{u}}(t), \boldsymbol{v}^{h}\right)\right|^{1 / 2}\right)
\end{aligned}
$$

Then, if $\|\beta\|_{L^{\infty}\left(\Gamma_{3}\right)}\left(\|\mu\|_{L^{\infty}\left(\Gamma_{3}\right)}+1\right)$ is sufficiently small, we have

(3.14) $\left\|\dot{\boldsymbol{u}}(t)-\dot{\boldsymbol{u}}^{h}(t)\right\|_{V}$

$$
\leq c\left(\left\|\dot{\boldsymbol{u}}(t)-\boldsymbol{v}^{h}\right\|_{V}+\left\|\boldsymbol{u}_{0}-\boldsymbol{u}_{0}^{h}\right\|_{V}+\int_{0}^{t}\left\|\dot{\boldsymbol{u}}(r)-\dot{\boldsymbol{u}}^{h}(r)\right\|_{V} d r+\left|R\left(t ; \dot{\boldsymbol{u}}(t), \boldsymbol{v}^{h}\right)\right|^{1 / 2}\right) .
$$

Notice that $\boldsymbol{v}^{h}$ is arbitrary in the above inequality. Using the Gronwall's inequality, we obtain

$$
\begin{aligned}
& \max _{t \in[0, T]}\left\|\dot{\boldsymbol{u}}(t)-\dot{\boldsymbol{u}}^{h}(t)\right\|_{V} \\
\leq & c\left\|\boldsymbol{u}_{0}-\boldsymbol{u}_{0}^{h}\right\|_{V}+c \max _{t \in[0, T]} \inf _{\boldsymbol{v}^{h} \in V^{h}}\left(\left\|\dot{\boldsymbol{u}}(t)-\boldsymbol{v}^{h}\right\|_{V}+\left|R\left(t ; \dot{\boldsymbol{u}}(t), \boldsymbol{v}^{h}\right)\right|^{1 / 2}\right) .
\end{aligned}
$$

The following estimate for $\boldsymbol{\sigma}-\boldsymbol{\sigma}^{h}$ follows directly from (3.8) and (3.15).

$$
\begin{gathered}
\max _{t \in[0, T]}\left\|\boldsymbol{\sigma}(t)-\boldsymbol{\sigma}^{h}(t)\right\|_{Q} \leq c\left\|\boldsymbol{u}_{0}-\boldsymbol{u}_{0}^{h}\right\|_{V}+\left\|\left(I_{Q}-\mathcal{P}_{Q^{h}}\right) \boldsymbol{\sigma}\right\|_{C([0, T] ; Q)} \\
+c \max _{t \in[0, T]} \inf _{\boldsymbol{v}^{h} \in V^{h}}\left(\left\|\dot{\boldsymbol{u}}(t)-\boldsymbol{v}^{h}\right\|_{V}+\left|R\left(t ; \dot{\boldsymbol{u}}(t), \boldsymbol{v}^{h}\right)\right|^{1 / 2}\right)
\end{gathered}
$$

Summarizing, we have shown the following result.

THEOREM 3.1. Let $(\boldsymbol{u}, \boldsymbol{\sigma})$ and $\left(\boldsymbol{u}^{h}, \boldsymbol{\sigma}^{h}\right)$ be the solutions of the problems $\mathbf{P}$ and $\mathbf{P}^{h}$ respectively. Assume the conditions stated in Theorem 2.1. Then if $\|\beta\|_{L^{\infty}\left(\Gamma_{3}\right)}\left(\|\mu\|_{L^{\infty}\left(\Gamma_{3}\right)}+1\right)$ is sufficiently small, the estimates (3.15) and (3.16) hold.

In order to give more concrete results based on (3.15) and (3.16), we now briefly specify the finite dimensional spaces $V^{h}$ and $Q^{h}$ via the finite element method. Details can be found in [4]. For simplicity, we assume that $\Omega$ is polygonal. Let $\mathcal{T}^{h}$ be a regular finite element partition of the domain $\Omega$. Here we use linear elements for the space $V^{h}$ and piecewise constants for $Q^{h}$.

To perform our convergence analysis, we need the following density result from [25].

Lemma 3.2. Assume that $X$ is a Banach space, $X_{0} \subset X$ is a dense subspace of $X$. Then $C\left([0, T] ; X_{0}\right)$ is dense in $C([0, T] ; X)$.

Now we are ready to give convergence and error analysis of the spatially discrete solution for the problem $\mathbf{P}$.

TheOREM 3.3. Keep all the assumptions in THEOREM 3.1. Assume the initial approximation $\boldsymbol{u}_{0}^{h} \in V^{h}$ is chosen such that

$$
\left\|u_{0}-u_{0}^{h}\right\|_{V} \rightarrow 0, \quad \text { as } \quad h \rightarrow 0
$$

Then

$$
\max _{t \in[0, T]}\left(\left\|\dot{\boldsymbol{u}}(t)-\dot{\boldsymbol{u}}^{h}(t)\right\|_{V}+\left\|\boldsymbol{\sigma}(t)-\boldsymbol{\sigma}^{h}(t)\right\|_{V}\right) \rightarrow 0 \quad \text { as } h \rightarrow 0
$$


Moreover, if

(3.19) $u \in C^{1}\left([0, T] ; V \cap\left(H^{2}(\Omega)\right)^{d} \cap\left(H^{2}\left(\Gamma_{3}\right)\right)^{d}\right), \quad \boldsymbol{\sigma} \in C\left([0, T] ;\left(H^{1}(\Omega)\right)^{d \times d}\right)$,

and

$$
\left\|u_{0}-u_{0}^{h}\right\|_{V} \leq c h
$$

then we have the optimal order error estimate

$$
\max _{t \in[0, T]}\left(\left\|\dot{\boldsymbol{u}}(t)-\dot{\boldsymbol{u}}^{h}(t)\right\|_{V}+\left\|\boldsymbol{\sigma}(t)-\boldsymbol{\sigma}^{h}(t)\right\|_{V}\right) \leq c h .
$$

Proof. Since $V \cap\left(H^{2}(\Omega)\right)^{d}$ and $\left(H^{1}(\Omega)\right)^{d \times d} \cap Q$ are dense in $V$ and $Q$ respectively, then by Lemma 3.2, $C\left([0, T] ; V \cap\left(H^{2}(\Omega)\right)^{d}\right)$ and $C\left([0, T] ;\left(H^{1}(\Omega)\right)^{d \times d} \cap Q\right)$ are dense in $C([0, T] ; V)$ and $C([0, T] ; Q)$ respectively. Therefore, $\forall \varepsilon>0$, there exist $\tilde{\boldsymbol{u}} \in$ $C\left([0, T] ; V \cap\left(H^{2}(\Omega)\right)^{d}\right)$ and $\tilde{\boldsymbol{\sigma}} \in C\left([0, T] ;\left(H^{1}(\Omega)\right)^{d \times d} \cap Q\right)$ such that

$$
\|\dot{u}-\tilde{u}\|_{C([0, T] ; V)} \leq \varepsilon, \quad\|\sigma-\tilde{\sigma}\|_{C([0, T] ; Q)} \leq \varepsilon .
$$

Let $\Pi^{h} \boldsymbol{v} \in V^{h}$ be the piecewise linear interpolant of $\boldsymbol{v} \in V \cap\left(H^{2}(\Omega)\right)^{d}$. Then (cf. [4])

$$
\left\|\boldsymbol{v}-\Pi^{h} \boldsymbol{v}\right\|_{V} \leq c h\|\boldsymbol{v}\|_{\left(H^{2}(\Omega)\right)^{d}} .
$$

Now we estimate the term $\left|R\left(t ; \dot{\boldsymbol{u}}(t), \boldsymbol{v}^{h}\right)\right|$ in (3.15) defined by (3.9). Recalling the assumption (2.11) and the trace theorem (3.12), we can show that

$$
\left|R\left(t ; \dot{u}(t), \boldsymbol{v}^{h}\right)\right| \leq c\left\|\dot{u}(t)-\boldsymbol{v}^{h}\right\|_{V} .
$$

By (3.22) and (3.23), we obtain from (3.15) that

$$
\begin{aligned}
\max _{t \in[0, T]}\left\|\dot{u}(t)-\dot{u}^{h}(t)\right\|_{V} & \leq c\left\|u_{0}-u_{0}^{h}\right\|_{V}+c \max _{t \in[0, T]} \inf _{v^{h} \in V^{h}}\left\|\dot{u}(t)-v^{h}\right\|_{V}^{1 / 2} \\
& \leq c\left\|u_{0}-u_{0}^{h}\right\|_{V}+c \varepsilon^{1 / 2}+c \max _{t \in[0, T]}\left\|\tilde{u}(t)-\Pi^{h} \tilde{u}(t)\right\|_{V}^{1 / 2} \\
& \leq c\left\|u_{0}-u_{0}^{h}\right\|_{V}+c \varepsilon^{1 / 2}+c h^{1 / 2}\|\tilde{u}\|_{C\left([0, T] ;\left(H^{2}(\Omega)\right)^{d}\right)^{1 / 2}} .
\end{aligned}
$$

Using the assumption (3.17), we conclude from the above estimate the convergence of $\dot{\boldsymbol{u}}^{h}$ to $\dot{\boldsymbol{u}}$.

Observe that the error bound of (3.16) differs from that of (3.15) only in one extra term $\left\|\left(I_{Q}-\mathcal{P}_{Q^{h}}\right) \boldsymbol{\sigma}\right\|_{C([0, T] ; Q)}$. Thus the convergence of $\boldsymbol{\sigma}^{h}$ to $\boldsymbol{\sigma}$ follows from the estimate

$$
\begin{aligned}
\left\|\left(I_{Q}-\mathcal{P}_{Q^{h}}\right) \boldsymbol{\sigma}\right\|_{C([0, T] ; Q)} & \leq\|\boldsymbol{\sigma}-\tilde{\boldsymbol{\sigma}}\|_{C([0, T] ; Q)}+\max _{t \in[0, T]} \inf _{\boldsymbol{q}^{h} \in Q^{h}}\left\|\tilde{\boldsymbol{\sigma}}-\boldsymbol{q}^{h}\right\|_{Q} \\
& \leq \varepsilon+c h\|\tilde{\boldsymbol{\sigma}}\|_{C\left([0, T] ;\left(H^{1}(\Omega)\right)^{d \times d}\right)},
\end{aligned}
$$

by using (3.2), (3.22) and finite element interpolation error estimates ([4]).

We now prove the error estimate (3.21) under the solution regularity condition (3.19) and the assumption (3.20). We re-estimate the term

$$
\begin{aligned}
R\left(t ; \dot{\boldsymbol{u}}(t), \boldsymbol{v}^{h}\right)= & \int_{\Omega}\left(\dot{\boldsymbol{\sigma}}(t) \cdot \boldsymbol{\varepsilon}\left(\boldsymbol{v}^{h}-\dot{\boldsymbol{u}}(t)\right)-\boldsymbol{f}(t) \cdot\left(\boldsymbol{v}^{h}-\dot{\boldsymbol{u}}(t)\right)\right) d x-\int_{\Gamma_{2}} \boldsymbol{g}(t) \cdot\left(\boldsymbol{v}^{h}-\dot{\boldsymbol{u}}(t)\right) d s \\
& +\int_{\Gamma_{3}} \beta\left|\dot{u}_{\nu}(t)\right|\left(\mu\left(\left|\boldsymbol{v}_{\tau}^{h}-\boldsymbol{v}^{*}\right|-\left|\dot{\boldsymbol{u}}_{\tau}(t)-\boldsymbol{v}^{*}\right|\right)+v_{\nu}^{h}-\dot{u}_{\nu}(t)\right) d s .
\end{aligned}
$$


Integrate by parts,

$$
\begin{aligned}
R\left(t ; \dot{\boldsymbol{u}}(t), \boldsymbol{v}^{h}\right)= & \left.\int_{\Omega}(-\operatorname{div} \boldsymbol{\sigma}(t)-\boldsymbol{f}(t)) \cdot\left(\boldsymbol{v}^{h}-\dot{\boldsymbol{u}}(t)\right)\right) d x \\
& +\int_{\Gamma} \boldsymbol{\sigma}(t) \boldsymbol{\nu} \cdot\left(\boldsymbol{v}^{h}-\dot{\boldsymbol{u}}(t)\right) d s-\int_{\Gamma_{2}} \boldsymbol{g}(t) \cdot\left(\boldsymbol{v}^{h}-\dot{\boldsymbol{u}}(t)\right) d s \\
& +\int_{\Gamma_{3}} \beta\left|\dot{u}_{\nu}(t)\right|\left(\mu\left(\left|\boldsymbol{v}_{\tau}^{h}-\boldsymbol{v}^{*}\right|-\left|\dot{u}_{\tau}(t)-\boldsymbol{v}^{*}\right|\right)+v_{\nu}^{h}-\dot{u}_{\nu}(t)\right) d s .
\end{aligned}
$$

Using the equilibrium equation and the boundary conditions on $\Gamma_{1} \Gamma_{2}$, we have

$$
\begin{gathered}
R\left(t ; \dot{\boldsymbol{u}}(t), \boldsymbol{v}^{h}\right)= \\
\int_{\Gamma_{3}}\left[\boldsymbol{\sigma}(t) \boldsymbol{\nu} \cdot\left(\boldsymbol{v}^{h}-\dot{\boldsymbol{u}}(t)\right)+\beta\left|\dot{u}_{\nu}(t)\right|\left(\mu\left(\left|\boldsymbol{v}_{\tau}^{h}-\boldsymbol{v}^{*}\right|-\left|\dot{\boldsymbol{u}}_{\tau}(t)-\boldsymbol{v}^{*}\right|\right)+v_{\nu}^{h}-\dot{u}_{\nu}(t)\right)\right] d s
\end{gathered}
$$

Therefore,

$$
\begin{aligned}
& \left|R\left(t ; \dot{\boldsymbol{u}}(t), \boldsymbol{v}^{h}\right)\right| \\
\leq & \left(\|\boldsymbol{\sigma}(t) \boldsymbol{\nu}\|_{\left(L^{2}\left(\Gamma_{3}\right)\right)^{d}}+\|\beta\|_{L^{\infty}\left(\Gamma_{3}\right)}\left(\|\mu\|_{L^{\infty}\left(\Gamma_{3}\right)}+1\right)\left\|\dot{u}_{\nu}(t)\right\|_{L^{2}\left(\Gamma_{3}\right)}\right)\left\|\dot{\boldsymbol{u}}(t)-\boldsymbol{v}^{h}\right\|_{\left(L^{2}\left(\Gamma_{3}\right)\right)^{d}},
\end{aligned}
$$

which with the aid of the result (3.12) yields

$$
\begin{gathered}
\left|R\left(t ; \dot{\boldsymbol{u}}(t), \boldsymbol{v}^{h}\right)\right| \leq\left(\|\boldsymbol{\sigma}\|_{C\left([0, T] ;\left(H^{1}(\Omega)\right)^{d \times d}\right)}+\|\beta\|_{L^{\infty}\left(\Gamma_{3}\right)}\left(\|\mu\|_{L^{\infty}\left(\Gamma_{3}\right)}+1\right)\|\dot{\boldsymbol{u}}\|_{C([0, T] ; V)}\right) \\
\cdot\left\|\dot{\boldsymbol{u}}(t)-\boldsymbol{v}^{h}\right\|_{\left(L^{2}\left(\Gamma_{3}\right)\right)^{d}}
\end{gathered}
$$

Hence from the estimate (3.15), it follows that

$$
\begin{aligned}
& \max _{t \in[0, T]}\left\|\dot{\boldsymbol{u}}(t)-\dot{\boldsymbol{u}}^{h}(t)\right\|_{V} \\
\leq & c\left\|\boldsymbol{u}_{0}-\boldsymbol{u}_{0}^{h}\right\|_{V}+c \max _{t \in[0, T]} \inf _{\boldsymbol{v}^{h} \in V^{h}}\left(\left\|\dot{\boldsymbol{u}}(t)-\boldsymbol{v}^{h}\right\|_{V}+\left\|\dot{\boldsymbol{u}}(t)-\boldsymbol{v}^{h}\right\|_{\left(L^{2}\left(\Gamma_{3}\right)\right)^{d}}^{1 / 2}\right) .
\end{aligned}
$$

With the regularity condition (3.19), using the interpolation results, we have

$$
\begin{aligned}
& \left\|\boldsymbol{u}(t)-\Pi^{h} \boldsymbol{u}(t)\right\|_{V} \leq c h\|\boldsymbol{u}(t)\|_{\left(H^{2}(\Omega)\right)^{d}} \\
& \left\|\boldsymbol{u}(t)-\Pi^{h} \boldsymbol{u}(t)\right\|_{\left(L^{2}\left(\Gamma_{3}\right)\right)^{d}} \leq c h^{2}\|\boldsymbol{u}(t)\|_{\left(H^{2}\left(\Gamma_{3}\right)\right)^{d}} \\
& \left\|\left(I_{Q}-\mathcal{P}_{Q^{h}}\right) \boldsymbol{\sigma}(t)\right\|_{Q} \leq c h\|\boldsymbol{\sigma}(t)\|_{\left(H^{1}(\Omega)\right)^{d \times d}}
\end{aligned}
$$

for all $t \in[0, T]$. Then the error estimate (3.21) follows from (3.16), (3.20) and (3.24). 口

4. Fully discrete approximation. Now we consider a fully discrete approximation of the problem $P$. In addition to the finite dimensional spaces $V^{h}$ and $Q^{h}$ introduced in the last subsection, we need a partition of the time interval $[0, T]: 0=$ $t_{0}<t_{1}<\cdots<t_{N}=T$. We denote the step-size $k_{n}=t_{n}-t_{n-1}$ for $n=1, \ldots, N$. We allow non-uniform partition of the time interval, and denote $k=\max _{n} k_{n}$ the maximal step-size. For a continuous function $w(t)$, we use the notation $w_{n}=w\left(t_{n}\right)$. For a sequence $\left\{w_{n}\right\}_{n=0}^{N}$, we denote $\Delta w_{n}=w_{n}-w_{n-1}$ for the difference, and $\delta w_{n}=\Delta w_{n} / k_{n}$ the corresponding divided difference. In this section, no summation is implied over the repeated index $n$. 
Then a fully discrete approximation method based on a backward Euler scheme is the following.

Problem $\mathbf{P}^{h k}$. Find the displacement field $\boldsymbol{u}^{h k}=\left\{\boldsymbol{u}_{n}^{h k}\right\}_{n=0}^{N} \subset V^{h}$ and the stress field $\sigma^{h k}=\left\{\sigma_{n}^{h k}\right\}_{n=0}^{N} \subset Q^{h}$, such that

$$
u_{0}^{h k}=u_{0}^{h}
$$

and for $n=1, \ldots, N$,

(4.2) $\boldsymbol{\sigma}_{n}^{h k}=\mathcal{P}_{Q^{h}} A\left(\varepsilon\left(\delta \boldsymbol{u}_{n}^{h k}\right)\right)+\mathcal{P}_{Q^{h}} G\left(\varepsilon\left(\boldsymbol{u}_{n-1}^{h k}\right)\right) \quad$ in $\Omega$,

(4.3) $\left\langle\sigma_{n}^{h k}, \varepsilon\left(\boldsymbol{v}^{h}-\delta \boldsymbol{u}_{n}^{h k}\right)\right\rangle+j\left(\delta \boldsymbol{u}_{n}^{h k}, \boldsymbol{v}^{h}\right)-j\left(\delta \boldsymbol{u}_{n}^{h k}, \delta \boldsymbol{u}_{n}^{h k}\right) \geq L\left(t_{n} ; \boldsymbol{v}^{h}-\delta \boldsymbol{u}_{n}^{h k}\right) \forall \boldsymbol{v}^{h} \in V^{h}$.

Using the argument technique of [21], it can be shown that the fully discrete problem $\mathbf{P}^{h k}$ has a unique solution if $\|\beta\|_{L^{\infty}\left(\Gamma_{3}\right)}\left(\|\mu\|_{L^{\infty}\left(\Gamma_{3}\right)}+1\right)$ is sufficiently small.

We now derive some error estimates for the numerical solution. We will use the notations $\boldsymbol{u}_{n}=\boldsymbol{u}\left(t_{n}\right), \dot{\boldsymbol{u}}_{n}=\dot{\boldsymbol{u}}\left(t_{n}\right)$ and $\sigma_{n}=\boldsymbol{\sigma}\left(t_{n}\right)$. We use (2.12) and (2.13) at $t=t_{n}$ and take $\boldsymbol{v}=\delta \boldsymbol{u}_{n}^{h k}$ to obtain

$$
\begin{aligned}
& \left\langle A\left(\varepsilon\left(\dot{\boldsymbol{u}}_{n}\right)\right)+G\left(\varepsilon\left(\boldsymbol{u}_{n}\right)\right), \boldsymbol{\varepsilon}\left(\delta \boldsymbol{u}_{n}^{h k}-\dot{\boldsymbol{u}}_{n}\right)\right\rangle+j\left(\dot{\boldsymbol{u}}_{n}, \delta \boldsymbol{u}_{n}^{h k}\right)-j\left(\dot{\boldsymbol{u}}_{n}, \dot{\boldsymbol{u}}_{n}\right) \\
\geq & L\left(t_{n} ; \delta \boldsymbol{u}_{n}^{h k}-\dot{\boldsymbol{u}}_{n}\right) .
\end{aligned}
$$

Substituting (4.2) into (4.3), we get

$$
\begin{aligned}
& \left\langle A\left(\varepsilon\left(\delta \boldsymbol{u}_{n}^{h k}\right)\right)+G\left(\varepsilon\left(\boldsymbol{u}_{n-1}^{h k}\right)\right), \varepsilon\left(\boldsymbol{v}^{h}-\delta \boldsymbol{u}_{n}^{h k}\right)\right\rangle+j\left(\delta \boldsymbol{u}_{n}^{h k}, \boldsymbol{v}^{h}\right)-j\left(\delta \boldsymbol{u}_{n}^{h k}, \delta \boldsymbol{u}_{n}^{h k}\right) \\
\geq & L\left(t ; \boldsymbol{v}^{h}-\delta \boldsymbol{u}_{n}^{h k}\right) .
\end{aligned}
$$

Adding (4.4) and (4.5) with rearrangement of the terms, we obtain

$$
\begin{aligned}
& \left\langle A\left(\varepsilon\left(\dot{\boldsymbol{u}}_{n}\right)\right)-A\left(\varepsilon\left(\delta \boldsymbol{u}_{n}^{h k}\right)\right), \varepsilon\left(\dot{\boldsymbol{u}}_{n}-\delta \boldsymbol{u}_{n}^{h k}\right)\right\rangle \\
\leq & -\left\langle G\left(\varepsilon\left(\boldsymbol{u}_{n}\right)\right)-G\left(\varepsilon\left(\boldsymbol{u}_{n-1}^{h k}\right)\right), \boldsymbol{\varepsilon}\left(\dot{\boldsymbol{u}}_{n}-\delta \boldsymbol{u}_{n}^{h k}\right)\right\rangle \\
& +\left\langle A\left(\varepsilon\left(\delta \boldsymbol{u}_{n}^{h k}\right)\right)-A\left(\varepsilon\left(\dot{\boldsymbol{u}}_{n}\right)\right)+G\left(\varepsilon\left(\boldsymbol{u}_{n-1}^{h k}\right)\right)-G\left(\varepsilon\left(\boldsymbol{u}_{n}\right)\right), \boldsymbol{\varepsilon}\left(\boldsymbol{v}^{h}-\dot{\boldsymbol{u}}_{n}\right)\right\rangle \\
& +R\left(t_{n} ; \dot{\boldsymbol{u}}_{n}, \boldsymbol{v}^{h}\right)+D\left(\dot{\boldsymbol{u}}_{n}, \delta \boldsymbol{u}_{n}^{h k}, \boldsymbol{v}^{h}\right),
\end{aligned}
$$

where the quantities $R\left(t_{n} ; \dot{\boldsymbol{u}}_{n}, \boldsymbol{v}^{h}\right)$ and $D\left(\dot{\boldsymbol{u}}_{n}, \delta \boldsymbol{u}_{n}^{h k}, \boldsymbol{v}^{h}\right)$ are defined in (3.9) and (3.10). Using the conditions (2.9) and (2.10), we obtain

$$
\begin{aligned}
& \left\|\varepsilon\left(\dot{u}_{n}-\delta \boldsymbol{u}_{n}^{h k}\right)\right\|_{Q}^{2} \\
\leq & c\left\|\varepsilon\left(\boldsymbol{u}_{n}-\boldsymbol{u}_{n-1}^{h k}\right)\right\|_{Q}\left\|\varepsilon\left(\dot{\boldsymbol{u}}_{n}-\delta \boldsymbol{u}_{n}^{h k}\right)\right\|_{Q} \\
& +c\left(\left\|\varepsilon\left(\dot{\boldsymbol{u}}_{n}-\delta \boldsymbol{u}_{n}^{h k}\right)\right\|_{Q}+\left\|\varepsilon\left(\boldsymbol{u}_{n}-\boldsymbol{u}_{n-1}^{h k}\right)\right\|_{Q}\right)\left\|\varepsilon\left(\dot{\boldsymbol{u}}_{n}-\boldsymbol{v}^{h}\right)\right\|_{Q} \\
& +c\left(\left|R\left(t_{n} ; \dot{\boldsymbol{u}}_{n}, \boldsymbol{v}^{h}\right)\right|+\left|D\left(\dot{\boldsymbol{u}}_{n}, \delta \boldsymbol{u}_{n}^{h k}, \boldsymbol{v}^{h}\right)\right|\right) .
\end{aligned}
$$

Then we have

$$
\begin{aligned}
& \left\|\varepsilon\left(\dot{\boldsymbol{u}}_{n}-\delta \boldsymbol{u}_{n}^{h k}\right)\right\|_{Q} \\
\leq & c\left(\left\|\varepsilon\left(\boldsymbol{u}_{n}-\boldsymbol{u}_{n-1}^{h k}\right)\right\|_{Q}+\left\|\varepsilon\left(\dot{\boldsymbol{u}}_{n}-\boldsymbol{v}^{h}\right)\right\|_{Q}+\left|R\left(t_{n} ; \dot{\boldsymbol{u}}_{n}, \boldsymbol{v}^{h}\right)\right|^{1 / 2}+\left|D\left(\dot{\boldsymbol{u}}_{n}, \delta \boldsymbol{u}_{n}^{h k}, \boldsymbol{v}^{h}\right)\right|^{1 / 2}\right) .
\end{aligned}
$$

Korn's inequality (2.8) can be applied here to yield

(4.6) $\left\|\dot{\boldsymbol{u}}_{n}-\delta \boldsymbol{u}_{n}^{h k}\right\|_{V}$

$$
\leq c\left(\left\|\boldsymbol{u}_{n}-\boldsymbol{u}_{n-1}^{h k}\right\|_{V}+\left\|\dot{\boldsymbol{u}}_{n}-\boldsymbol{v}^{h}\right\|_{V}+\left|R\left(t_{n} ; \dot{\boldsymbol{u}}_{n}, \boldsymbol{v}^{h}\right)\right|^{1 / 2}+\left|D\left(\dot{\boldsymbol{u}}_{n}, \delta \boldsymbol{u}_{n}^{h k}, \boldsymbol{v}^{h}\right)\right|^{1 / 2}\right) .
$$


From the estimate (3.13) and the result (3.12),

$$
\begin{aligned}
& \left|D\left(\dot{\boldsymbol{u}}_{n}, \delta \boldsymbol{u}_{n}^{h k}, \boldsymbol{v}^{h}\right)\right|^{1 / 2} \\
\leq & c\|\beta\|_{L^{\infty}\left(\Gamma_{3}\right)}^{1 / 2}\left(\|\mu\|_{L^{\infty}\left(\Gamma_{3}\right)}+1\right)^{1 / 2}\left\|\dot{u}_{n}-\delta u_{n}^{h k}\right\|_{V}+c\left\|\dot{u}_{n}-v^{h}\right\|_{V} .
\end{aligned}
$$

Using this estimate in (4.6), we obtain

$$
\begin{aligned}
\left\|\dot{\boldsymbol{u}}_{n}-\delta \boldsymbol{u}_{n}^{h k}\right\|_{V} \leq & c\|\beta\|_{L^{\infty}\left(\Gamma_{3}\right)}^{1 / 2}\left(\|\mu\|_{L^{\infty}\left(\Gamma_{3}\right)}+1\right)^{1 / 2}\left\|\dot{\boldsymbol{u}}_{n}-\delta \boldsymbol{u}_{n}^{h k}\right\|_{V} \\
& +c\left\|\boldsymbol{u}_{n}-\boldsymbol{u}_{n-1}^{h k}\right\|_{V}+c \inf _{\boldsymbol{v}^{h} \in V^{h}}\left\{\left\|\dot{\boldsymbol{u}}_{n}-\boldsymbol{v}^{h}\right\|_{V}+\left|R\left(t_{n} ; \dot{\boldsymbol{u}}_{n}, \boldsymbol{v}^{h}\right)\right|^{1 / 2}\right\} .
\end{aligned}
$$

If $\|\beta\|_{L^{\infty}\left(\Gamma_{3}\right)}\left(\|\mu\|_{L^{\infty}\left(\Gamma_{3}\right)}+1\right)$ is sufficiently small, we have

$$
\begin{aligned}
& \left\|\dot{\boldsymbol{u}}_{n}-\delta \boldsymbol{u}_{n}^{h k}\right\|_{V} \\
\leq & c\left\|\boldsymbol{u}_{n}-\boldsymbol{u}_{n-1}^{h k}\right\|_{V}+c \inf _{\boldsymbol{v}^{h} \in V^{h}}\left\{\left\|\dot{\boldsymbol{u}}_{n}-\boldsymbol{v}^{h}\right\|_{V}+\left|R\left(t_{n} ; \dot{\boldsymbol{u}}_{n}, \boldsymbol{v}^{h}\right)\right|^{1 / 2}\right\} .
\end{aligned}
$$

Write

$$
\boldsymbol{u}_{n}-\boldsymbol{u}_{n-1}^{h k}=\boldsymbol{u}_{0}-\boldsymbol{u}_{0}^{h}+\boldsymbol{u}_{n}-\boldsymbol{u}_{n-1}+\sum_{j=1}^{n-1} k_{j}\left(\delta \boldsymbol{u}_{j}-\dot{\boldsymbol{u}}_{j}\right)+\sum_{j=1}^{n-1} k_{j}\left(\dot{\boldsymbol{u}}_{j}-\delta \boldsymbol{u}_{j}^{h k}\right) .
$$

Then

$$
\begin{aligned}
\left\|\boldsymbol{u}_{n}-\boldsymbol{u}_{n-1}^{h k}\right\|_{V} \leq & \left\|\boldsymbol{u}_{0}-\boldsymbol{u}_{0}^{h}\right\|_{V}+\left\|\boldsymbol{u}_{n}-\boldsymbol{u}_{n-1}\right\|_{V} \\
& +\sum_{j=1}^{n-1} k_{j}\left\|\dot{\boldsymbol{u}}_{j}-\delta \boldsymbol{u}_{j}\right\|_{V}+\sum_{j=1}^{n-1} k_{j}\left\|\dot{\boldsymbol{u}}_{j}-\delta \boldsymbol{u}_{j}^{h k}\right\|_{V}
\end{aligned}
$$

Hence from (4.7), we get

$$
\begin{array}{r}
\text { (4.9) }\left\|\dot{\boldsymbol{u}}_{n}-\delta \boldsymbol{u}_{n}^{h k}\right\|_{V} \leq c\left\|\boldsymbol{u}_{0}-\boldsymbol{u}_{0}^{h}\right\|_{V}+c\left\|\boldsymbol{u}_{n}-\boldsymbol{u}_{n-1}\right\|_{V}+c \sum_{j=1}^{n-1} k_{j}\left\|\dot{\boldsymbol{u}}_{j}-\delta \boldsymbol{u}_{j}\right\|_{V} \\
+c \inf _{\boldsymbol{v}^{h} \in V^{h}}\left\{\left\|\dot{\boldsymbol{u}}_{n}-\boldsymbol{v}^{h}\right\|_{V}+\left|R\left(t_{n} ; \dot{\boldsymbol{u}}_{n}, \boldsymbol{v}^{h}\right)\right|^{1 / 2}\right\}+c \sum_{j=1}^{n-1} k_{j}\left\|\dot{\boldsymbol{u}}_{j}-\delta \boldsymbol{u}_{j}^{h k}\right\|_{V} .
\end{array}
$$

To proceed further, we need the following result.

LEMMA 4.1. Assume $\left\{g_{n}\right\}_{n=1}^{N}$ and $\left\{e_{n}\right\}_{n=1}^{N}$ are two sequences of non-negative numbers, satisfying

$$
e_{n} \leq c g_{n}+c \sum_{j=1}^{n-1} k_{j} e_{j}, \quad n=1, \ldots, N
$$

Then

$$
e_{n} \leq c\left(g_{n}+\sum_{j=1}^{n-1} k_{j} g_{j}\right), \quad n=1, \ldots, N
$$


Therefore,

$$
\max _{1 \leq n \leq N} e_{n} \leq c \max _{1 \leq n \leq N} g_{n}
$$

Proof. Denote

$$
E_{n}=\sum_{j=1}^{n} k_{j} e_{j}
$$

Then

$$
e_{n} \leq c g_{n}+c E_{n-1}, \quad n=1, \ldots, N
$$

Now

$$
E_{n}-E_{n-1}=k_{n} e_{n} \leq c k_{n} g_{n}+c k_{n} E_{n-1}
$$

which implies

$$
E_{n}-\left(1+c k_{n}\right) E_{n-1} \leq c k_{n} g_{n} \quad n=1, \ldots, N .
$$

We introduce a sequence of numbers $\left\{z_{n}\right\}_{n=0}^{N}$ by setting $z_{0}=1$ and

$$
z_{n}=\prod_{j=1}^{n}\left(1+c k_{j}\right), \quad 1 \leq n \leq N .
$$

Using the inequalities

$$
1 \leq 1+c k_{j} \leq e^{c k_{j}}, \quad j=1, \ldots, N
$$

we have the following bounds:

$$
1 \leq \prod_{i=j+1}^{N}\left(1+c k_{i}\right) \leq e^{c\left(T-t_{j}\right)}, \quad j=1, \ldots, N
$$

Now with the above notations, the inequality (4.13) can be rewritten as

$$
\frac{E_{n}}{z_{n}}-\frac{E_{n-1}}{z_{n-1}} \leq \frac{c k_{n} g_{n}}{z_{n}}
$$

A simple induction argument gives us

$$
E_{n} \leq c z_{n} \sum_{j=1}^{n} k_{j} \frac{g_{j}}{z_{j}}=c \sum_{j=1}^{n} k_{j} \prod_{i=j+1}^{n}\left(1+c k_{i}\right) g_{j}
$$

which can be combined with (4.12) and (4.14) to yield (4.10). The inequality (4.11) follows easily from (4.10).

Applying LEMmA 4.1 to the inequality (4.9), we obtain the following estimate,

$$
\begin{aligned}
& \max _{1 \leq n \leq N}\left\|\dot{\boldsymbol{u}}_{n}-\delta \boldsymbol{u}_{n}^{h k}\right\|_{V} \\
\leq & c\left\{\left\|\boldsymbol{u}_{0}-\boldsymbol{u}_{0}^{h}\right\|_{V}+\sum_{n=1}^{N-1} k_{n}\left\|\delta \boldsymbol{u}_{n}-\dot{\boldsymbol{u}}_{n}\right\|_{V}\right\}+c \max _{1 \leq n \leq N}\left\|\boldsymbol{u}_{n}-\boldsymbol{u}_{n-1}\right\|_{V} \\
& +c \max _{1 \leq n \leq N} \inf _{\boldsymbol{v}^{h} \in V^{h}}\left\{\left\|\dot{\boldsymbol{u}}_{n}-\boldsymbol{v}^{h}\right\|_{V}+\left|R\left(t_{n} ; \dot{\boldsymbol{u}}_{n}, \boldsymbol{v}^{h}\right)\right|^{1 / 2}\right\}
\end{aligned}
$$


Now we turn to bound $\sigma_{n}-\sigma_{n}^{h k}$. Subtracting (4.2) from (2.12) at $t=t_{n}$, we have $\boldsymbol{\sigma}_{n}-\boldsymbol{\sigma}_{n}^{h k}=\left(I_{Q}-\mathcal{P}_{Q^{h}}\right) \boldsymbol{\sigma}_{n}+\mathcal{P}_{Q^{h}}\left[A\left(\varepsilon\left(\dot{\boldsymbol{u}}_{n}\right)\right)-A\left(\varepsilon\left(\delta \boldsymbol{u}_{n}^{h k}\right)\right)+G\left(\varepsilon\left(\boldsymbol{u}_{n}\right)\right)-G\left(\varepsilon\left(\boldsymbol{u}_{n-1}^{h k}\right)\right)\right]$, which, by the assumptions (2.9), (2.10) and the property (3.2), yields

$$
\left\|\boldsymbol{\sigma}_{n}-\boldsymbol{\sigma}_{n}^{h k}\right\|_{Q} \leq\left\|\left(I_{Q}-\mathcal{P}_{Q^{h}}\right) \boldsymbol{\sigma}_{n}\right\|_{Q}+c\left\|\dot{\boldsymbol{u}}_{n}-\delta \boldsymbol{u}_{n}^{h k}\right\|_{V}+c\left\|\boldsymbol{u}_{n}-\boldsymbol{u}_{n-1}^{h k}\right\|_{V}
$$

Recalling (4.8), we then get the estimate

$$
\begin{aligned}
& \text { (4.16) } \max _{1 \leq n \leq N}\left\|\boldsymbol{\sigma}_{n}-\boldsymbol{\sigma}_{n}^{h k}\right\|_{V} \\
& \leq c\left\{\left\|\boldsymbol{u}_{0}-\boldsymbol{u}_{0}^{h}\right\|_{V}+\sum_{n=1}^{N-1} k_{n}\left\|\delta \boldsymbol{u}_{n}-\dot{\boldsymbol{u}}_{n}\right\|_{V}\right\}+\left\|\left(I_{Q}-\mathcal{P}_{Q^{h}}\right) \boldsymbol{\sigma}\right\|_{C([0, T] ; Q)} \\
& \quad+c \max _{1 \leq n \leq N}\left\|\boldsymbol{u}_{n}-\boldsymbol{u}_{n-1}\right\|_{V}+c \max _{1 \leq n \leq N} \inf _{\boldsymbol{v}^{h} \in V^{h}}\left\{\left\|\dot{\boldsymbol{u}}_{n}-\boldsymbol{v}^{h}\right\|_{V}+\left|R\left(t_{n} ; \dot{\boldsymbol{u}}_{n}, \boldsymbol{v}^{h}\right)\right|^{1 / 2}\right\} .
\end{aligned}
$$

Summarizing, we have shown the following result.

THEOREM 4.2. Let $(\boldsymbol{u}, \boldsymbol{\sigma})$ and $\left\{\left(\boldsymbol{u}_{n}^{h k}, \boldsymbol{\sigma}_{n}^{h k}\right)\right\}_{n=1}^{N}$ be the solutions of the problems $\mathbf{P}$ and $\mathbf{P}^{h k}$ respectively. Assume the conditions stated in Theorem 2.1. Then if $\|\beta\|_{L^{\infty}\left(\Gamma_{3}\right)}\left(\|\mu\|_{L^{\infty}\left(\Gamma_{3}\right)}+1\right)$ is sufficiently small, the estimates (4.15) and (4.16) hold.

The following result (cf. [25]) is needed for convergence analysis.

Lemma 4.3. Assume that $X$ is a Banach space. Then $C^{\infty}([0, T] ; X)$ is dense in $C^{1}([0, T] ; X)$.

TheOREM 4.4. Keep all the assumptions in THEOREM 4.2. Assume the initial approximation $u_{0}^{h} \in V^{h}$ is chosen such that

$$
\left\|\boldsymbol{u}_{0}-\boldsymbol{u}_{0}^{h}\right\|_{V} \rightarrow 0, \quad \text { as } \quad h \rightarrow 0
$$

Then

$$
\max _{1 \leq n \leq N}\left(\left\|\dot{\boldsymbol{u}}_{n}-\delta \boldsymbol{u}_{n}^{h k}\right\|_{V}+\left\|\boldsymbol{\sigma}_{n}-\boldsymbol{\sigma}_{n}^{h k}\right\|_{V}\right) \rightarrow 0 \quad \text { as } h, k \rightarrow 0 .
$$

Moreover, if

$$
\left\{\begin{array}{c}
\boldsymbol{u} \in C^{1}\left([0, T] ; V \cap\left(H^{2}(\Omega)\right)^{d} \cap\left(H^{2}\left(\Gamma_{3}\right)\right)^{d}\right) \cap W^{2,1}(0, T ; V) \\
\boldsymbol{\sigma} \in C\left([0, T] ;\left(H^{1}(\Omega)\right)^{d \times d}\right)
\end{array}\right.
$$

and

$$
\left\|u_{0}-u_{0}^{h}\right\|_{V} \leq c h
$$

then we have the optimal order error estimate

$$
\max _{1 \leq n \leq N}\left(\left\|\dot{u}_{n}-\delta \boldsymbol{u}_{n}^{h k}\right\|_{V}+\left\|\boldsymbol{\sigma}_{n}-\boldsymbol{\sigma}_{n}^{h k}\right\|_{V}\right) \leq c(h+k)
$$

Proof. First we show the convergence result (4.18). Most terms on the right-hand sides of the estimates (4.15) and (4.16) can be handled in much the same way as those 
similar terms on the right-hand side of the estimate (3.16), as was done in the proof of Theorem 3.3. Here it is enough for us to estimate the two terms

$$
\sum_{n=1}^{N-1} k_{n}\left\|\delta u_{n}-\dot{u}_{n}\right\|_{V} \quad \text { and } \max _{1 \leq n \leq N}\left\|u_{n}-u_{n-1}\right\|_{V} .
$$

By Lemma $4.3, C^{\infty}([0, T] ; V)$ is dense in $C^{1}([0, T] ; V)$. Hence $\forall \varepsilon>0$, there exists $\boldsymbol{w} \in C^{\infty}([0, T] ; V)$ such that

$$
\|u-w\|_{C^{1}([0, T] ; V)} \leq \varepsilon .
$$

Since

$$
\begin{aligned}
\left\|\delta \boldsymbol{u}_{n}-\dot{\boldsymbol{u}}_{n}\right\|_{V} & \leq\left\|\delta\left(\boldsymbol{u}_{n}-\boldsymbol{w}_{n}\right)-\left(\dot{\boldsymbol{u}}_{n}-\dot{\boldsymbol{w}}_{n}\right)\right\|_{V}+\left\|\delta \boldsymbol{w}_{n}-\dot{\boldsymbol{w}}_{n}\right\|_{V} \\
& \leq \varepsilon+\left\|\frac{1}{k} \int_{t_{n-1}}^{t_{n}}\left(\dot{\boldsymbol{w}}(t)-\dot{\boldsymbol{w}}_{n}\right) d t\right\|_{V} \\
& \leq \varepsilon+\int_{t_{n-1}}^{t_{n}}\|\ddot{\boldsymbol{w}}(t)\|_{V} d t, \quad n=1, \ldots, N-1,
\end{aligned}
$$

we have

$$
\sum_{n=1}^{N-1} k_{n}\left\|\delta u_{n}-\dot{u}_{n}\right\|_{V} \leq T \varepsilon+k\|\ddot{\boldsymbol{w}}\|_{L^{1}(0, T ; V)},
$$

where $\ddot{\boldsymbol{w}}(t)$ denotes the second-order derivative of $\boldsymbol{w}(t)$ with respect to the time variable $t$. It is easily seen that

$$
\max _{1 \leq n \leq N}\left\|u_{n}-u_{n-1}\right\|_{V} \leq k\|\dot{u}\|_{C([0, T] ; V)} .
$$

Thus we have the convergence (4.18).

Under the regularity condition (4.19), we have

$$
\sum_{n=1}^{N-1} k_{n}\left\|\delta u_{n}-\dot{u}_{n}\right\|_{V} \leq k\|\ddot{u}\|_{L^{1}(0, T ; V)} .
$$

The estimate (4.21) can be proved in much the same way as the estimate (3.21) was proved in the previous section.

\section{REFERENCES}

[1] R. A. Adams, Sobolev Spaces, Academic Press, New York, 1975.

[2] K. T. Andrews, A. Klarbring, M. Shillor, and S. Wright, A dynamic contact problem with friction and wear, Int. J. Engng. Sci., 35 (1997), pp. 1291-1309.

[3] J. J. Bikerman, Thermodynamics, adhesion, and sliding friction, J. Lubr. Technol., 92 (1970), pp. 243-247.

[4] P. G. Ciarlet, The Finite Element Method for Elliptic Problems, North Holland, Amsterdam, 1978.

[5] G. Duvaut and J. L. Lions, Inequalities in Mechanics and Physics, Springer-Verlag, Berlin, 1976.

[6] R. GLowinski, Numerical Methods for Nonlinear Variational Problems, Springer-Verlag, New York, 1984. 
[7] R. Glowinski, J.-L. Lions, and R. TRÉmolì̀res, Numerical Analysis of Variational Inequalities, North-Holland, Amsterdam, 1981.

[8] W. HAN AND B. D. REDDY, Computational plasticity: the variational basis and numerical analysis, Computational Mechanics Advances, 2 (1995), pp. 283-400.

[9] W. HAN AND B. D. REDDY, Convergence of approximations to the primal problem in plasticity under conditions of minimal regularity, Numer. Math., 87 (2000), pp. 283-315.

[10] W. HAN AND B. D. REDDY, Plasticity: Mathematical Theory and Numerical Analysis, SpringerVerlag, New York, 1999.

[11] I. HlavÁČEK, J. Haslinger, J. NECĂs, AND J. LovíšEK, Solution of Variational Inequalities in Mechanics, Springer-Verlag, New York, 1988.

[12] N. Kikuchi And J. T. Oden, Contact Problems in Elasticity: A Study of Variational Inequalities and Finite Element Methods, SIAM, Philadelphia, 1988.

[13] B. E. KLAMECKI, Wear-an entropy production model, Wear, 58 (1980), pp. 325-330.

[14] B. E. KLAMECKI, A thermodynamic model of friction, Wear, 63 (1980), pp. 113-120.

[15] B. E. KLAMECKI, An entropy-based model of plastic deformation energy dissipation in sliding, Wear, 96 (1984), pp. 319-329.

[16] M. M. KHRUSChov, Principles of abrasive wear, Wear, 28 (1974), pp. 69-88.

[17] J. NeČAs AND I. HLAVAČEK, Mathematical Theory of Elastic and Elastoplastic Bodies: An Introduction, Elsevier, Amsterdam, 1981.

[18] M. Raous, M. Jean, And J. J. Moreau (eds.), Contact Mechanics, Plenum Press, New York, 1995.

[19] M. ROchDI, M. SHILloR, AND M. SOFONEA, Quasistatic viscoelastic contact with normal compliance and friction, Journal of Elasticity, 51 (1998), pp. 105-126.

[20] M. Rochdi, M. Shillor, AND M. Sofonea, A quasistatic contact problem with directional friction and damped response, Applicable Analysis, 68 (1998), pp. 409-422.

[21] M. Shillor AND M. SofoneA, A quasistatic viscoelastic contact problem with friction, Int. J. Engng. Sci., 38 (2000), pp. 1517-1533.

[22] N. Strömberg, Continuum Thermodynamics of Contact, Friction and Wear, Ph.D. Thesis, Linköping University, Sweden, 1995.

[23] N. Strömberg, L. Johansson, AND A. Klarbring, Derivation and analysis of a generalized standard model for contact friction and wear, Int. J. Solids Structures, 33 (1996), pp. 1817-1836.

[24] D. TABOR, Wear-a critical synoptic view, J. Lubr. Technol., 99 (1977), pp. 387-395.

[25] E. ZEIDLER, Nonlinear Functional Analysis and its Applications, Volume II/A: Linear Monotone Operators, Springer-Verlag, New York, 1985. 